\title{
Improving post-impact care-a form of injury prevention
}

\author{
David Yates
}

An analysis of Western European data suggests that the number of deaths attributable to trauma is in decline. There is an assumption that this fall in mortality is a direct consequence of improvements to the environment and, to a lesser extent, to changes in behaviour. Examples include initiatives that have been termed primary prevention such as traffic segregation and product design and those termed secondary prevention that include seat belts and helmet laws. However, the direct causal relationship between the introduction of such measures and subsequent change in the mortality rate is difficult to prove. The link with the changing patterns of disability is often even more elusive.

Closer examination of the available statistics indicates that this general trend towards fewer trauma deaths is not equally distributed across society. Mortality rates are falling more slowly in the young adult population and in deprived sections of the community. The effect of so-called host factors, that is pre-existing medical problems in those who are injured may, in part, explain this phenomenon. These host factors are, of course, particularly evident in the older population, but the well known association between deprivation and poor health means that the less privileged members of society of all ages are at double jeopardy. Their circumstances mean that they are more likely to sustain injury and the higher incidence of host factors coupled with inadequate support networks may impair their ability to make a satisfactory recovery.

There is, however, an additional problem affecting all sections of society. The size of the trauma epidemic is unnecessarily increased by the inefficiency of the health service's response. Pre-hospital care is improving but still of variable quality. Sometimes it is over zealous and frequently it is inadequately linked with the hospital phase of resuscitation. The concept of a trauma system is still poorly understood in many countries and the rehabilitation services are usually very under-resourced. Improving these components of the care of the injured has been termed tertiary prevention.

\section{Department of \\ Accident and \\ Emergency Medicine, \\ Hope Hospital, Salford \\ M6 8HD}

Correspondence to: Professor Yates (e-mail: dyates@fs1.ho.man.ac.uk).

Evidence of inadequate trauma care

Deficiencies in the system of trauma care in the area around San Francisco were first identified by West et al in 1979. ${ }^{1}$ They showed that in a county in which patients were taken to the nearest of 31 departments offering an emergency service, the outcome, in terms of patient survival, was markedly worse than it was in the adjacent county where all the severely injured were taken to a single hospital. In the single trauma unit only $1 \%$ of deaths were considered by a panel of experts to have been preventable, whereas in the other county between $28 \%$ and $73 \%$ of deaths were considered preventable. The causes of death were said to be easily remediable-for example ruptured spleen and extradural haemorrhage. The authors considered that the improved outcome observed in the trauma unit was a result of a combination of earlier recognition of life threatening injuries and their prompt treatment by experienced surgeons and anaesthetists.

This alarming revelation has been both substantiated and challenged by many authors over the last 20 years. Initial and perhaps naive optimism for the potential benefits of trauma centres has been replaced by a recognition of the importance of trauma systems, with their emphasis on integration of care, from the scene of the incident through to rehabilitation services. This concept has been promoted by a working party on the Management of Patients with Major Injuries which reported to the Royal College of Surgeons of England in $1988 .^{2}$ The effective components of a trauma system and the limitations to its implementation can be summarised as follows.

(A) Public access to emergency services

- Indiscriminate use leads to inappropriate response.

- Need for prioritisation of calls by trained dispatchers.

(B) Rapid deployment of paramedic crews

- Relative infrequency of major trauma results in decay of skills.

- Need for frequent refresher courses and integration of paramedics with others involved in trauma care.

(C) Minimum on-scene time

- Tendency to delay at scene and to use learnt skills even when not needed.

- Need to know when not to stay and play.

(D) Controlled but rapid transfer to hospital
- Nearest hospital is not necessarily the best hospital.

- Need to plan regional trauma services to reduce interhospital transfers. 
(E) Integrated hospital response

- Inexperienced reception staff may fail to recognise treatment priorities.

- Need to harmonise response at senior level between relevant specialties.

(F) Early rehabilitation and discharge planning

- Tendency to attend to medical detail and overlook the big picture.

- Need to involve wide spectrum of rehabilitation and community care specialists as soon as possible.

\section{Widening the network of care}

Opportunities to further extend the effectiveness of the emergency healthcare system have been promoted in the UK in recent years. Translated into terms relevant to the management of trauma, the concept has included first aiders, pharmacists, and primary healthcare teams as well as those involved in the more traditional components of the trauma care system from paramedic through to intensivist. ${ }^{3}$ There is a further challenge however-to promote a comprehensive reduction in the scale of the trauma epidemic by encouraging these lay and professional carers to embrace both primary and secondary prevention. Historically, clinicians have been reluctant to become involved in public health matters, but there is a great opportunity for them to use their public profile and experience to influence policy makers. The Australasian College of Surgeons has been particularly effective in helping to reduce road trauma deaths by promoting both improved standards of care and road safety legislation.

Collecting data and auditing effectiveness Continuing demographic and social changes and the recognition that care after impact in the UK may not be optimal has led to a demand for better information systems, and in particular, an emphasis on the importance of denominator information to complement the more usually acquired numerator statistics. While the concept of exposure rates is well known to public health physicians, clinicians usually limit their analyses to patients who come to their attention in hospital. Many reports even exclude those who die en route to hospital, and virtually all analyses conducted by those responsible for the organisation of trauma services are unable to comment on the rate of exposure of their injured population. It is therefore very difficult to get a balanced view of the impact of any preventive measure, whether it be primary, secondary, or tertiary.

In 1980 Champion et al proposed that patient management should not be driven by dogma but by data. ${ }^{4}$ They advocated the use of injury scoring systems that allowed the many different types of injuries sustained by trauma victims to be characterised numerically. The abbreviated injury scale (AIS) was developed to help engineers design safer aircraft and cars but is now used to assess the severity of most types of injury. It scores injuries from 0-6 roughly corresponding with increasing severity.
Although initially consensus derived - that is to say developed by a group of experts without recourse to data-the AIS score has been widely adopted and now is considered to be an international standard. ${ }^{5}$ The AIS can be used to describe a patient with multiple injuries using a technique described by Baker et al to give the injury severity score (ISS). ${ }^{6}$ By combining this anatomically based score with a physiological scoring system (the revised trauma score) the overall threat to life of an injured patient can be quantified. ${ }^{7}$ This is the TRISS methodology (an acronym derived from trauma score and ISS), now widely used to calculate the probability of survival. The probability of survival of a group of patients can then be compared with their observed mortality to give a measure of the effectiveness of care (standardised W statistic). The W statistic can be used as a performance indicator between institutions or within institutions over time. ${ }^{8} \mathrm{~A}$ number of challenges have been made to this TRISS methodology. It is labour intensive in terms of data collection and analysis and does not adequately measure burns or some types of major brain injuries or asphyxia. However it remains popular and has the advantage of consistency for international comparisons.

Audit programmes using this type of scoring system were developed initially in North America and, since 1988, have been used in the UK. The first report of the Major Trauma Outcome Study in the UK was published in 1992. ${ }^{9}$ It drew attention to the significant variation between actual survival rates in $33 \mathrm{UK}$ hospitals and those computed from severity matched controls treated in some North American hospital trauma systems. Delays were identified in the initial resuscitation of seriously injured patients, there was a lack of involvement of senior staff and delays in the provision of urgent surgery. Additionally, the study revealed wide discrepancies between participating hospitals in the UK. Recent international comparisons have confirmed similar variations between centres across Europe. Importantly for those concerned with injury prevention, the differences are sufficiently great to complicate the interpretation of national statistics of accidental death. These data are usually understood to reflect the incidence of trauma with the implicit assumption that its consequences are inevitably the same whatever the circumstances. The finding of significant variations in mortality rate in different centres, for the same injury, means that these national data are unlikely to reflect variations in road safety alone-they will also reflect variations in post-impact care.

It is therefore not surprising that there is increasing interest in trauma scoring from public health physicians and those responsible for the development of healthcare systems. The second series of epidemiologically based needs assessment reviews by the Wessex Institute includes an extensive section on accident and emergency departments. In their analysis of outcomes the authors write "....the introduction of the Major Trauma Outcome Study system for auditing performance with major trauma is a com- 
mendable development and purchasers will probably wish to stipulate its use...". ${ }^{10}$

In 1997 the Major Trauma Outcome Study expanded its remit to include population based analyses and placed greater emphasis on epidemiological research. The renamed UK Trauma Audit and Research Network now receives data from the majority of major trauma receiving hospitals in England and Wales and is supported by a grant from the Central Health Outcomes Unit of the Department of Health. Collaborative work is conducted with other centres across Europe. While tertiary prevention remains its chief concern there is an awareness of the importance of establishing dialogue with those whose focus is primary and secondary prevention. This is the best way to ensure that those responsible for resource allocation are able to identify the most appropriate use of public funds.

The quality of the data accumulated by the UK Trauma Audit and Research Network is variable. There is constant tension between the demands for data accuracy and the financial and organisational strains that these demands impose on the clinical service. However, with over 100000 cases on the database there are now opportunities to make longitudinal analyses of the effectiveness of trauma care in the UK over a number of years. While the results must be treated with caution (because initiatives to ensure that the data are representative of the catchment population are not yet robust) the conclusions do merit consideration and are relevant to the debate about the contribution of medical care to the reduction in the number of injury related deaths. One specific example of this type of calculation is given here.

\section{Falling death rate in children and young adults}

Roberts et al used the database of the Trauma Audit and Research Network to examine changing case fatality rates over a seven year period. ${ }^{11}$ Case fatality was defined as the proportion of patients with an ISS of 16 or more who died as a result of their injuries. The analysis was restricted to patients with blunt trauma under the age of 25. Logistic regression modelling was used to examine the temporal trend in the probability of death with adjustments made for age, ISS, and revised trauma score. The expected mortality was calculated as the sum of the probability of death for each patient as predicted by TRISS methodology. At the beginning of the study only 30 hospitals were contributing significant numbers of cases whereas at the end, in 1995, over 100 hospitals were participating. Calculations were therefore carried out using all data from all hospitals and, separately, from the original cohort of 30 hospitals.

Over the study period 16710 injured patients aged 0-24 years fulfilled criteria for inclusion in the Trauma Network database and $20 \%$ (3394) had an ISS of 16 or more, signifying major injury. Ninety five per cent of this latter group (3230) had sustained blunt trauma and had been treated at any of 122 centres in
England and Wales. Most (71\%) had been involved in a road crash but $17 \%$ had been injured in a fall. There was no significant change in these proportions during the study period. Similarly there was no change in the median ISS.

Analysis of the survival rate over the seven year period showed an almost linear decline in the log odds of death. Using the previously described TRISS methodology and W statistic calculations to control for variations in the severity of injury, the expected mortality rate was calculated for each year. The ratio of the observed to the expected death rates showed a similar decline over the seven year period. Subgroup analysis showed that this decline was greatest for children younger than 5 years. When all these calculations were repeated on data only from the 30 hospitals that had been participating in this audit process from the start, the results were essentially unchanged.

Plotting these results as estimated odds of death in each year, relative to the baseline of 1989 , the first year of the study, it was apparent that there was a clear downward trend that could not be explained by differences in severity of injury or age. These results suggest therefore, that reductions in hospital case fatality have made an important contribution to the objective of reducing accidental deaths in the UK. They do not, of course, provide any evidence about the changing patterns of disability. Measurement of disability and impairment is fraught with difficulty but it is generally accepted that medical estimates underrepresent the true size of the problem. ${ }^{12}$ Attempts to use scoring systems to measure the effectiveness of post-impact care in reducing the rate of disability have yet to meet with success. ${ }^{13}$ However its importance is recognised by all who are concerned with the measurement of injury severity as a first step in developing a comprehensive injury prevention strategy. ${ }^{14}$

\section{Conclusions and recommendations}

There are now sufficient data available to suggest that injury prevention strategies will be incomplete if they fail to take account of the potential for further improvements in both resuscitation and rehabilitation. Clinicians responsible for the development of enhanced trauma care systems should therefore work more closely with public health physicians and others to ensure that all possible methods of reducing trauma mortality and morbidity rates are addressed.

- The improvement of post-impact care should be accepted as a form of injury prevention.

- Social deprivation, already acknowledged as an important factor in the aetiology of trauma, must also be taken into account when assessing the effectiveness of postimpact care.

- Informed debate depends upon accurate data. These must take into account expo- 
sure rates, the previous health of the population studied, and the process of care.

- Most studies have been concerned with avoidable mortality, but it is probable that the extent and duration of post-traumatic disability can also be reduced by more effective care. There is an urgent need to improve disability scoring techniques so that this problem can be more accurately assessed.

- Currently available statistics are often incorrectly interpreted as reflecting the incidence of trauma.

Recognition that they also reflect the process of care has implications for those responsible for the allocation of funds between public health and clinical services.

1 West J, Trunkey DD, Lim RC. Systems of trauma care. Arch Surg 1979;114:455-60.

2 Royal College of Surgeons of England Working Party. Management of patients with major injuries. London: Royal College of Surgeons of England, 1988.

3 Emergency Care Project Steering Group. Opportunities in emergency healthcare. Milton Keynes: Anglia and Oxford Region, 1995.
4 Champion HR, Sacco WJ, Hannan JDS, et al. Assessment of injury severity. Crit Care Med 1980;8:201-8.

5 Huang LC, March JC. AIS and threat to life. Proceedings of the Association of Advanced Automotive Medicine 1978;22: 242-54

6 Baker SP, O'Neill B, Haddon W, et al. The injury severity score: a method for describing patients with multiple injuries and evaluating emergency care. $\mathcal{F}$ Trauma 1974;14: 187-96.

7 Boyd CR, Tolson MA, Copes WS. Evaluating trauma care: the TRISS method. F Trauma 1987;27:370-8.

8 Hollis S, Yates DW, Woodford M, et al. Standardised comparison of performance indicators in trauma: a new approach to case mix variation. F Trauma 1995;38:763-6.

9 Yates DW, Woodford M, Hollis S. Preliminary analysis of the care of injured patients in 33 British hospitals: first report of the UK Major Trauma Outcome Study. BMF 1992;305:737-40.

10 Williams B, Nicholl J, Brazier J. Accident and emergency departments. In: Stevens A, Raftery J, eds. Health care needs assessment. 2nd Series. Wessex Institute. Oxford: Radcliffe assessment. 2nd Series. Wessex

11 Roberts I, Campbell F, Hollis S, et al. Reducing accident death rates in children and young adults: the contribution of hospital care. BMF 1996;313:1239-41.

12 Galasko CSB, Murray P, Hodson M, et al. Long term disability following road traffic accidents. Crowthorne, Berkshire: Transport and Road Research Laboratory, 1986. Research report 59 .

13 Yates DW, Health DF, Mars E, et al. A system for measuring the severity of temporary and permanent disability after injury. Accid Anal Prev 1991;23:323-9.

14 European Transport Safety Council. A strategic road safety plan for the European Union. Brussels: ETSC (Rue du Cornet 34, Brussels, B 1040, Belgium), 1997: 42. 